\title{
Preface to the Special Issue on "Fatigue, Cyclic Deformation and Microstructure"
}

\author{
Yukichi UMAKOSHI and Masaharu KATO ${ }^{1)}$
}

Department of Materials Science and Engineering, Faculty of Engineering, Osaka University, Yamada-oka, Suita, Osaka-fu, 565 Japan. E-mail:umakoshi@mat.eng.osaka-u.ac.jp 1) Department of Innovative and Engineering Materials, Interdisciplinary Graduate School of Science and Engineering, Tokyo Institute of Technology, Nagatsuta, Midori-ku, Yokohama, Kanagawa-ken, 226 Japan. E-mail: kato@materia.titech.ac.jp

Since structural materials are often subjected to the repeated application of stresses or strains, knowledge of the plastic behavior under cyclic loading conditions is required to develop and improve mechanical properties for practical use. Research on the fatigue of engineering materials started about 150 years ago. The aspects of fatigue life, crack initiation, propagation and fracture behavior have been examined focusing on the development of alloy design and engineering approaches in industrial use. With the growing interest in advanced materials such as intermetallics and ceramics, topics of fatigue research continue to expand at a rapid rate.

In order to develop and improve the fatigue properties of conventional and advanced materials, basic research on fatigue phenomena is required. Cyclic hardening, fracture and fatigue should be understood as a result of the irreversible to-and-fro motion of dislocations and the formation of their peculiar deformation substructure during cyclic loading.

This special issue covers the state-of-the-art topics of cyclic deformation, fracture behavior and fatigue properties of not only conventional materials of steels and aluminium alloys but also advanced intermetallics of titanium aluminides and silicides. Three review papers which cover the fatigue properties of intermetallics, steels and eight contributed papers are presented. All manuscripts were critiqued by one technical reviewer and an editor. We would like to thank all the authors for their excellent papers. We would appreciate the good cooperation of the comments of the reviewers to improve the quality of the papers.

We extend our thanks to Prof. N. S. Stoloff, Prof. H. Mughrabi, Prof. H. J. Christ, Dr. O. Umezawa and Dr. K. Nagai for excellent review papers.

We hope that this special issue will provide engineers and scientists with the innovation of fatigue research. 